\title{
Influencia del raleo en el crecimiento, la condición hídrica y la respuesta fotosintética de Peumus boldus: pautas para la definición de su manejo silvicultural
}

\author{
Influence of thinning over growth, water potential and photosynthetic response of Peumus boldus: \\ guidelines for their silvicultural management proposal
}

\author{
Sergio Donoso ${ }^{\text {a*}}$, Karen Peña-Rojas a, Sergio Durán a , Cristian Pacheco a , Evelyn Galdames a, Claudia Espinoza a \\ *Autor de correspondencia: ${ }^{a}$ Universidad de Chile, Facultad de Ciencias Forestales y Conservación de la Naturaleza, \\ Santiago, Chile, sedonoso@uchile.cl
}

\section{SUMMARY}

Peumus boldus, an endemic tree species of central Chile, belongs to sclerophyllous forests. Its semi-xerophytes condition allows it to grow in places with low rainfall and poor soils. The current shrubby condition is due to the implemented management through a coppice regime. This management was proposed due to its low ability for seed regeneration and its high ability to sprout. Currently, the harvest of this species is mainly based on economic criteria, with a lacking of productivity and technical criteria to define its silviculture. It is essential to understand the response of sprouts after interventions to improve its management. The aim of this study was to evaluate the response during the first two years of $P$. boldus coppices with different cutting intensities. A set of 23 strains was annually monitored in three levels of intervention: total, partial (50\% basal area) and without stools cuts (control). Predawn water potential, chlorophyll fluorescence, diameter growth and above-ground biomass accumulation were evaluated. Results showed that cuts improved the hydric status and this status was directly related to the cutting intensity of the strains. The same effect was observed in the production and growth of new shoots. Finalizing the evaluation period, accumulated biomass was $17.9 \%$ in strains with total harvest and $53.6 \%$ in those with partial harvest. These results raise doubts over the ability of $P$. boldus to correctly recover after biomass harvesting in short management periods. These results show that strains of $P$. boldus can be utilized in a better way with partial harvest. Further studies are needed to determine intensity and frequency of harvest.

Key words: sclerophyllous forests, silviculture, predawn water potential, sprouts, biomass.

\section{RESUMEN}

Peumus boldus, especie arbórea endémica de Chile central del bosque esclerófilo, por su condición semi-xerófita se desarrolla con bajas precipitaciones y suelos pobres. Actualmente presenta estado arbustivo, debido a manejo de monte bajo por su alta capacidad de rebrote y baja regeneración por semilla. La cosecha de $P$. boldus se basa principalmente en criterios económicos, faltando criterios productivos y técnicos para definir su silvicultura. Para mejorar el manejo actual, es fundamental comprender la respuesta del rebrote después de la cosecha. El objetivo del estudio fue evaluar la respuesta de cepas de $P$. boldus sometidas a diferentes intensidades de cosecha, durante los dos primeros años después de la corta, para establecer pautas de manejo silvicultural de la especie. Para ello se monitoreó anualmente un grupo de 23 cepas con tres niveles de cosecha: total, parcial ( $50 \%$ del área basal) y sin intervención (control). Se evaluaron las respuestas: hídrica, proceso fotosintético mediante fluorescencia, crecimiento en diámetro y acumulación de biomasa aérea. Los resultados mostraron que las cortas mejoraron el estado hídrico de las cepas en relación directa a la intensidad aplicada, además de incrementar la aparición y crecimiento de nuevos rebrotes. Al final del segundo año de evaluación, la biomasa acumulada fue del 17,9\% de la biomasa inicial en las cepas con cosecha total, y del 53,6 \% en las con cosecha parcial. Estos resultados muestran que cepas de $P$. boldus pueden ser manejadas de mejor forma, con una cosecha parcial. Nuevos estudios son necesarios para determinar la intensidad y periodicidad de la cosecha.

Palabras clave: bosque esclerófilo, silvicultura, potencial hídrico, rebrote, biomasa.

\section{INTRODUCCIÓN}

Desde un punto de vista práctico, considerando la silvicultura de las especies, las plantas pueden dividirse entre aquellas que rebrotan o no rebrotan. La capacidad de rebrotar permite sobrevivir a daños en su estructura aérea causada por perturbaciones como fuego, ramoneo o intervenciones silvícolas (Paula y Ojeda 2006). El rebrote genera troncos o vástagos secundarios en árboles como una respuesta inducida por algún daño o cambio drástico en las condiciones medioambientales circundantes permitiendo su rápida recuperación (Del Tredici 2001, López et al. 
2009). Esta es una forma eficiente de regenerar la biomasa perdida (Bellingham y Sparrow 2000), sobre todo cuando existen dificultades para la germinación y el reclutamiento de nuevas plantas a partir de semillas (Handavu et al. 2011). En conjunto con el establecimiento de nuevas plantas provenientes de semilla, el rebrote se considera como un mecanismo de regeneración en los ecosistemas mediterráneos después de perturbaciones (López et al. 2009). La principal ventaja del rebrote por sobre las plantas provenientes de semilla, es su sistema radical bien establecido (Handavu et al. 2011, Forrester et al. 2012), que le permite enfrentar de mejor manera la competencia por agua, considerado el principal factor de crecimiento, reproducción y sobrevivencia en clima mediterráneo (Vilà y Sardans 1999). Esta capacidad de rebrote les confiere una alta resistencia tanto a eventos naturales como antrópicos, aunque su efectividad puede disminuir con la edad de los árboles (Vesk 2006) o si la frecuencia de estas perturbaciones es muy alta (López et al. 2009).

Las regiones mediterráneas, presentes en latitudes medias entre los paralelos $30^{\circ}$ y $40^{\circ}$ norte y sur, como la Cuenca del Mediterráneo, California, Chile central, la región del Cabo en Sudáfrica y el suroeste y sur de Australia (Vilà y Sardans 1999), se caracterizan por presentar veranos secos con bajas precipitaciones, altas temperaturas, alta irradiación y altos niveles de déficit de presión de vapor de agua. Estos factores son considerados clave debido a su influencia sobre las respuestas fisiológicas de las plantas, que responderán de forma diferente a los distintos cambios ambientales (Ogaya y Peñuelas 2003) considerado especialmente importante para el crecimiento y distribución de especies en estas regiones. La escasez de agua puede afectar el crecimiento y supervivencia de las plantas de forma directa, influyendo sobre la capacidad fotosintética por el cierre estomático, o indirecta por la alteración de la difusión de nutrientes hacia la raíz, produciendo ambas, bajas tasas de crecimiento (Ogaya et al. 2003).

Conocer el tipo de árboles que presentan mayor probabilidad de rebrote y cuál es su comportamiento, es relevante para comprender la ecología del bosque y planificar su manejo (Salk y McMahon 2011). En este ámbito, el raleo es una intervención silvícola común en el manejo de bosques mediterráneos, el cual puede imitar perturbaciones de diferente magnitud dependiendo de la intensidad de raleo aplicado (López et al. 2009).

Peumus boldus Mol. (boldo), es una especie arbórea endémica de Chile inserta en el bosque esclerófilo (Rodríguez et al. 1983), que frecuentemente se encuentra como monte bajo, presentando varios troncos secundarios o vástagos desde el tocón o cepa original, debido a que históricamente ha sido cosechada para extraer sus hojas medicinales y producción de carbón (Donoso 1989). Según Rodríguez et al. (1983), boldo puede desarrollarse en diversas condiciones, creciendo en lugares de poca humedad y sobre suelos a menudo pedregosos, pudiendo adecuarse a condiciones de sequía más o menos fuertes por su carácter semi-xerófito. El manejo de esta especie a través de monte bajo se explica por el bajo porcentaje de germinación de semillas y la excelente capacidad de rebrote (Rodríguez et al. 1983, Del Fierro 1998) y fundamentada principalmente por criterios económicos, donde el principal producto es la hoja, que presenta una alta demanda (Vita 1993).

En la actualidad, la prescripción silvícola autorizada, indica una intensidad de cosecha inferior a $35 \%$ del área basal, y se puede volver a cosechar nuevamente la misma superficie después de cinco años. Normalmente por la facilidad de cosecha y calidad de las hojas, se vuelven a intervenir las cepas, previamente cosechadas. Sin embargo, no hay antecedentes asociados a la base ecológica y criterios técnicos, y productivos que sustenten esta práctica. Por ello, el estudio analiza la silvicultura que actualmente se aplica a la especie.

El objetivo del estudio es evaluar en cepas de $P$. boldus la respuesta en crecimiento y variables fisiológicas de dos intensidades de corta en comparación con un testigo, durante los primeros dos años después de la cosecha.

La hipótesis de trabajo es que mientras mayor sea la intensidad de corta, la condición hídrica de los árboles en verano es más favorable, la respuesta de crecimiento de rebrotes es mayor y que existe una baja capacidad de recuperar la biomasa cosechada en el corto plazo, por corresponder a una especie que crece en un ambiente con una marcada restricción hídrica estival. La pregunta a responder es: ¿la forma actual de aprovechamiento de boldo garantiza la sostenibilidad?

\section{MÉTODOS}

El fundo Loleo ( $33^{\circ} 26^{\prime} \mathrm{S}$ y $71^{\circ} 16^{\prime} \mathrm{O}$ ), situado en la provincia de Melipilla, región Metropolitana de Santiago, Chile, se inserta en un clima mediterráneo semiárido, con una precipitación media anual de $349,8 \mathrm{~mm}$ y un período seco, de siete a ocho meses, donde la precipitación mensual es menor a $50 \%$ de la evapotranspiración potencial mensual (Uribe et al. 2012). En el área de estudio, el suelo corresponde a un alfisol (serie Lo Vásquez, Ultic Haploxeralfs) originado a partir de material granítico y presenta una estructura franco arcillo arenosa en superficie y arcillosa en profundidad, con un contenido de gravilla de cuarzo que se incrementa en profundidad (CIREN 1996). El verano del año 2011, se trabajó en un área con pendientes menores a $10 \%$, donde se establecieron tres sectores de estudio ( $2.500 \mathrm{~m}^{2}$ cada uno) en los que se identificaron las especies arbóreas presentes y se midió en cada cepa el diámetro a la altura del pecho $(1,3 \mathrm{~m})$ (DAP) de todos los vástagos. Se seleccionaron y marcaron un total de 23 cepas de diferentes tamaños. Posteriormente (marzo), el bosque fue intervenido para la cosecha de hojas de boldo: dos sectores fueron cosechados y uno permaneció sin intervención.

El diseño del estudio incluyó tres tratamientos sobre las cepas: cosecha total de vástagos $(n=6)$, cosecha parcial de vástagos, extracción de aproximadamente $50 \%$ de 
los vástagos $(\mathrm{n}=6)$ y sin intervención (control) $(\mathrm{n}=11)$. Las cepas control poseían similares características en número de vástagos y área basal de las cepas intervenidas. Los vástagos de las cepas cosechadas parcialmente y las cepas control fueron marcados a la altura del DAP para realizar el monitoreo del crecimiento diametral sobre la misma sección en los dos años siguientes (2012-2013).

La respuesta fisiológica fue evaluada en los rebrotes y en los vástagos remanentes fue evaluada en cada cepa cada mes de febrero. Esta época del año fue seleccionada, ya que los árboles en la zona de estudio se han visto expuesto a un prolongado período con altas temperaturas y déficit hídrico estival. Se monitoreó la respuesta hídrica midiendo el potencial hídrico foliar ( $\Psi$ a), a pre-alba y a medio día, de una ramilla con hojas completamente desarrolladas de vástago adulto y/o de rebrote según el tipo de cepa. Para ello se usó una cámara de presión tipo Scholander (PMS Instruments, Model 1000, USA). Cada ramilla fue pesada en una balanza de precisión $0,001 \mathrm{~g}$ para obtener su peso fresco (Pf), luego hidratada en agua destilada por 24 horas en condiciones de oscuridad a $4{ }^{\circ} \mathrm{C}$, para obtener su peso hidratado $(\mathrm{Ph})$, y finalmente llevada a una estufa de aire forzado a $65^{\circ} \mathrm{C}$ hasta alcanzar peso constante, para obtener su peso seco (Ps). Los pesos antes mencionados fueron utilizados para el cálculo del contenido hídrico relativo de la hoja (CHRa \%) [1]:

$$
C H R a \%=\frac{P f-P s}{P h-P S} \times 100
$$

La respuesta del fotosistema II (PSII) fue monitoreada simultáneamente con la respuesta hídrica, mediante el rendimiento cuántico máximo (PSII) de la fluorescencia medida a pre-alba, junto con las variables: eficiencia fotoquímica del fotosistema II (Ф PSII), "quenching” fotoquímico de la fluorescencia (qP), "quenching" no fotoquímico de la fluorescencia y tasa relativa de transporte de electrones (ETR), medidas al medio día. La cuantificación de los parámetros de fluorescencia de las clorofilas se realizó con un fluorímetro modulado portátil Mini-PAM (Photosynthesis Yield Analyser, Walz, Effeltrich, Alemania) con el método descrito por Peña-Rojas et al. (2004) y Fleck et al. (2010). Este análisis permite caracterizar efectos de diferentes estreses ambientales como: temperatura, sequía y alta intensidad luminosa.

El crecimiento de las cepas fue evaluado en los vástagos remanentes y rebrotes. En los vástagos remanentes, previamente marcados en otoño de 2011, se midió el diámetro a la altura del pecho $(1,3 \mathrm{~m})(\mathrm{DAP})$ y el diámetro a la altura del tocón $(0,3 \mathrm{~m})$ (DAT), con una cinta diamétrica con precisión de $0,1 \mathrm{~cm}$, esto se repitió después de la cosecha en marzo de 2012 y 2013. En las cepas se contó el número de rebrotes por cepa y se midió el diámetro a la altura del cuello (DAC) con una cinta diamétrica con precisión de $0,1 \mathrm{~cm}$ y la altura con una cinta de distancia con precisión al centímetro, en los meses de marzo de 2012 y 2013.
La biomasa aérea en las cepas control y en los vástagos remanentes de las cepas con cosecha parcial, fue estimada con el modelo propuesto por Donoso y Durán (2007).

Para estimar la biomasa de rebrote, se obtuvo una muestra de 50 rebrotes seleccionados a partir de la distribución de frecuencia del DAC, los cuales fueron trasladados al laboratorio, donde fueron procesados y secados en estufa de aire forzado a $65^{\circ} \mathrm{C}$ hasta alcanzar peso constante. Luego se determinó el peso en una balanza de precisión $(0,01 \mathrm{~g})$. Con los pesos se ajustó un modelo de acumulación de biomasa de rebrotes, con el DAC como variable independiente y el peso seco como variable dependiente.

Se realizaron análisis de la varianza (ANDEVA) de medidas repetidas para comparar las respuestas entre los tratamientos (cosecha total, cosecha parcial y no intervenido) analizando: (i) respuesta hídrica y (ii) crecimiento. La respuesta del fotosistema II (PSII) fue analizada mediante un análisis de varianza con tres tratamientos: cepas con cosecha total, cepa con cosecha parcial y cepas no intervenidas. Las diferencias detectadas entre medias se analizaron con una prueba de comparaciones múltiples de Tukey $(P<0,05)$.

\section{RESULTADOS}

El bosque estudiado presentó un área basal total de 23,4 $\mathrm{m}^{2}$ ha-1, del cual 9,1 $\mathrm{m}^{2}$ ha $^{-1}$ corresponden a P. boldus $(39,1 \%)$, donde la densidad de cepas de boldo por hectárea varió entre 1.125 y 1.640 con una media de 1.363 cepas $\mathrm{ha}^{-1}$. Las cepas de boldo analizadas presentaron, al inicio del estudio, en promedio 9,5 vástagos por cepa y un área basal que varió entre 10 y $220 \mathrm{~cm}^{2}$.

El $\Psi$ a de rebrotes y vástagos remanentes, en las cepas de cosecha parcial, no presentaron diferencias significativas $(P=0,27)$ durante el estudio. El primer año de evaluación, las cepas con intervención parcial y total, presentaron un $\Psi$ a significativamente menos negativo $(P<0,01)$ que las cepas control en la evaluación realizada a pre-alba (figura 1). La evaluación de mediodía, sólo para las cepas con cosecha total, fue significativamente menos negativa a los otros tratamientos. El segundo año de evaluación la condición hídrica de las cepas en general fue menos negativa, debido a que el monto de precipitaciones del año $2012(375,2 \mathrm{~mm})$ fue superior al año $2011(254,5 \mathrm{~mm})$ (DGA 20141). En febrero de 2013, se presentaron diferencias significativas entre los tres tratamientos, repitiéndose el mismo patrón en la evaluación a pre-alba y a mediodía, donde la condición hídrica más favorable la presentaron las cepas con cosecha total y el valor más negativo de $\Psi a$ las cepas control (figura 1). La intensidad de la intervención no influyó significativamente sobre el CHRa de la hoja, aunque se observó una tendencia a la pérdida de agua en la medida que el $\Psi$ a fue más negativo.

Datos consultados de la estación "Ibacache Alto" UTM 287838 6292823, provincia de Melipilla, región Metropolitana. 

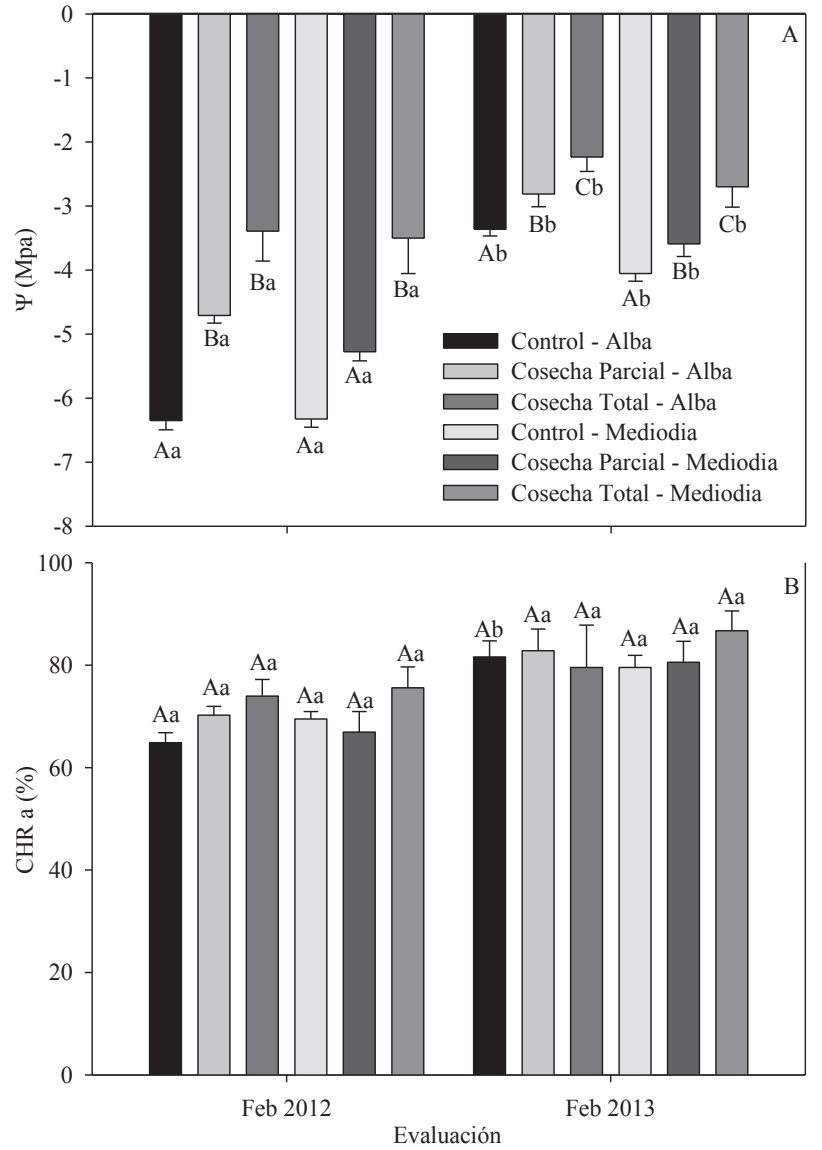

Figura 1. Efecto de diferentes tratamientos de corta en cepas de Peumus boldus sobre la condición hídrica (a pre-alba y a mediodía), en el verano de dos años consecutivos. A) Potencial hídrico ( $\Psi \mathrm{MPa}$ ) y B) Contenido hídrico relativo (CHRa \%). Las letras mayúsculas indican diferencias significativas entre tratamientos y las minúsculas entre meses de evaluación (Tukey, $P<0,05$ ).

Effect of different cut treatments applied to Peumus boldus coppice on water conditions (pre-dawn and noon), in the summer of two consecutive years. A) Water potential ( $\Psi, \mathrm{Mpa})$ and B) relative water content (CHRa \%). Capital letters indicate significant differences among treatments and lowercase letters indicate the differences among months of evaluation (Tukey, $P<0.05$ ).

Para el rendimiento cuántico máximo del PSII al alba, los resultados indican que no hay diferencias significativas entre los tratamientos de cosecha (figura 2), y también entre rebrotes y vástagos remanentes del tratamiento de corta parcial $(P<0,05)$, en los dos años evaluados.

Los parámetros obtenidos de las mediciones de fluorescencia de las clorofilas al mediodía, para evaluar el estado del PSII, Ф PSII, "quenching" fotoquímico de la fluorescencia, "quenching" no fotoquímico de la fluorescencia y la tasa relativa de transporte de electrones, no presentaron diferencias significativas entre los rebrotes y los vástagos adultos del tratamiento de corta parcial $(P<$ $0,05)$. Se observaron diferencias significativas al comparar los tratamientos de cosecha en "quenching" fotoquímico

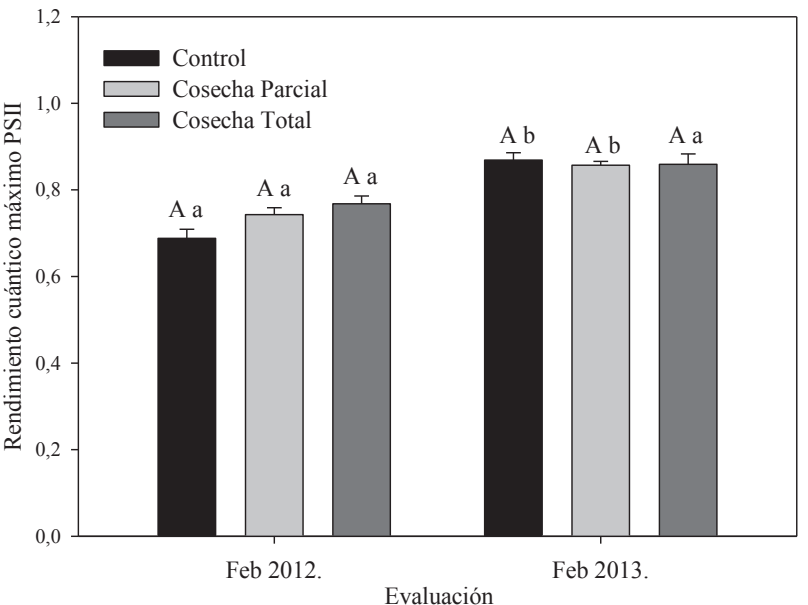

Figura 2. Respuesta de diferentes tratamientos de corta en cepas de Peumus boldus sobre el rendimiento cuántico máximo (PSII) de la fluorescencia al alba, en el verano de dos años consecutivos. Las letras mayúsculas indican diferencias significativas entre tratamientos y las minúsculas, entre meses de evaluación (Tukey, $P$ $<0,05)$.

Response of different cut treatments applied to Peumus bol$d u s$ coppice on yield maximum quantum (PSII) of the fluorescence at dawn, in the summer of two consecutive years. Capital letters indicate significant differences among treatments and lowercase letters among months of evaluation (Tukey, $P<0.05$ ).

de la fluorescencia, entre las cepas control y las sometidas a intervención parcial, siendo menor en el tratamiento control. El "quenching" no fotoquímico de la fluorescencia solo mostró una tendencia a disminuir en las cepas con cosecha parcial y $\Phi$ PSII una tendencia a disminuir en las cepas con cosecha total, sin ser significativas. En cambio, la tasa relativa de transporte de electrones presentó una tendencia no significativa a aumentar en las cepas con cosecha total (figura 3).

Después de un año de aplicado los tratamientos, la cantidad de rebrotes fue significativamente superior en las cepas con cosecha total respecto de las cepas con cosecha parcial, con 116 y 30 rebrotes en promedio respectivamente, presentando una alta variabilidad entre las cepas evaluadas. En el segundo año de crecimiento se produjo un aumento en el número de rebrotes total en las cepas con cosecha parcial (46 rebrotes) y una disminución en la cosecha total (90 rebrotes) con respecto al primer año, dejando de ser significativas las diferencias entre ambos tipos de cepa (figura 4). En las cepas control, sólo el segundo año aparecieron en promedio dos rebrotes por cepa.

El DAC promedio de los rebrotes no presentó diferencias significativas entre los tratamientos de cosecha, en ninguna de las dos evaluaciones. Las cepas control presentaron un DAC significativamente inferior el segundo año, pues en estas solo hubo nuevos brotes el segundo año de crecimiento (figura 4). El crecimiento en DAC el primer año fue de 3,2 y $3,5 \mathrm{~mm}_{\text {año }}{ }^{-1}$ y el segundo año fue de 4,0 

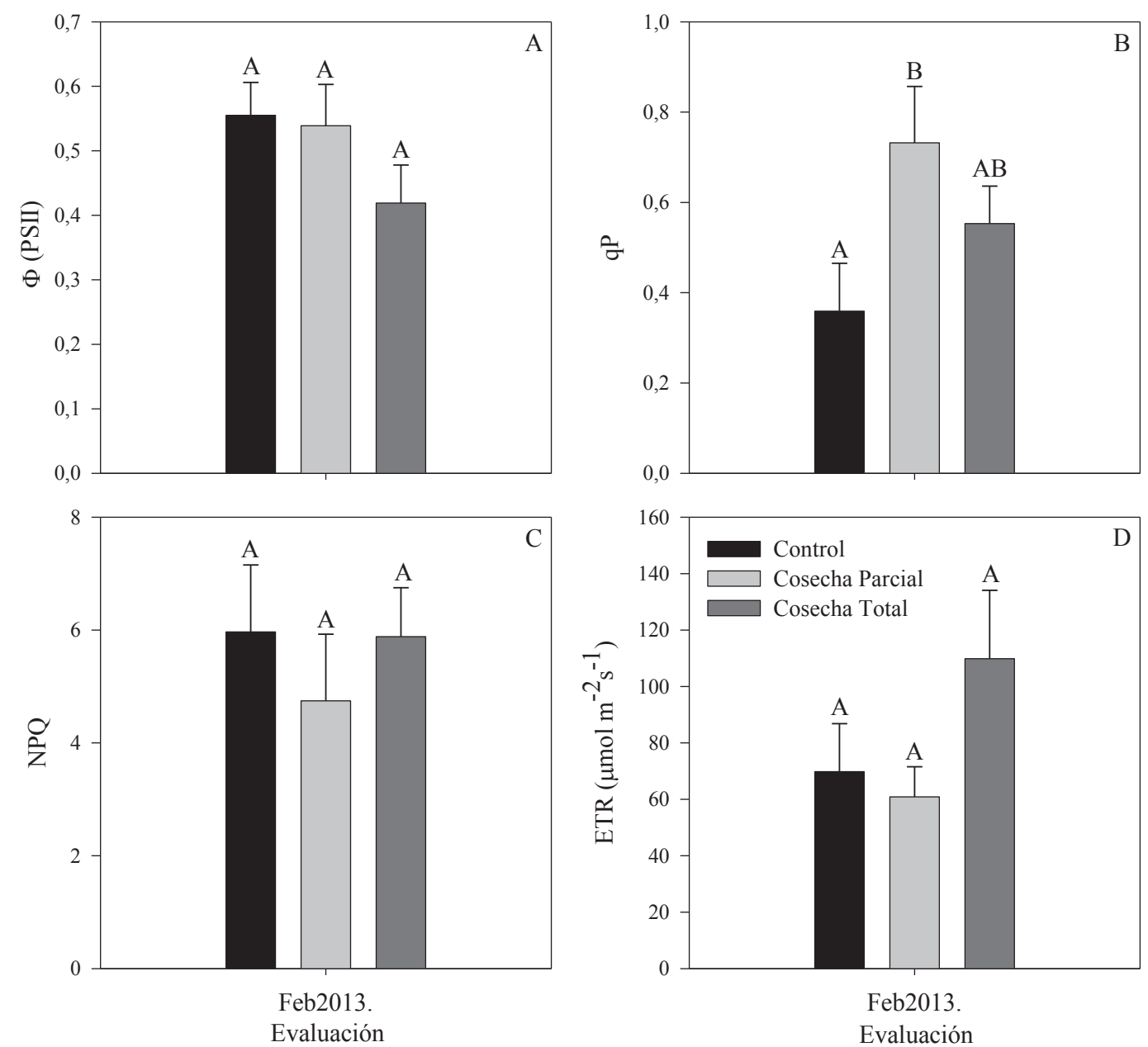

Figura 3. Respuesta de diferentes tratamientos de corta en cepas de Peumus boldus sobre el fotosistema II (PSII) al medio día, en el verano de dos años consecutivos. A) Eficiencia fotoquímica del fotosistema II (Ф PSII), B) "quenching" fotoquímico de la fluorescen-

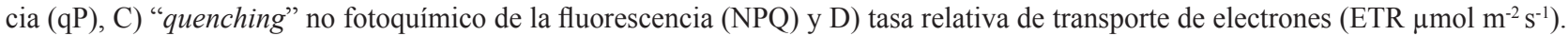
Las letras mayúsculas indican diferencias significativas entre tratamientos (Tukey, $P<0,05$ ).

Response of different cut treatments to Peumus boldus coppice on photosystem II (PSII) at noon, in the summer of two consecutive years. A) photochemical efficiency of photosystem II (PSII Ф), B) photochemical "quenching” of chlorophyll fluorescence (qP) C) Non-photochemical "quenching" of chlorophyll fluorescence (NPQ) and D) relative rate of electron transport (ETR $\left.\mu \mathrm{mol} \mathrm{m}^{-2} \mathrm{~s}^{-1}\right)$. Capital letters indicate significant differences among treatments (Tukey, $P<0.05$ ).

y 4,3 mm año $0^{-1}$, para las cepas con cosecha parcial y total, respectivamente. El crecimiento en altura tuvo un comportamiento similar con un crecimiento durante el primer año de $17,0 \mathrm{~cm}$ y $14,5 \mathrm{~cm}$ y el segundo año de 55,8 y $67,1 \mathrm{~cm}$, para las cepas con cosecha parcial y total, respectivamente (figura 4). Al estimar la biomasa de rebrotes con el modelo ajustado (figura 5), se determinó que en el primer año de evaluación las cepas con cosecha total presentaron un incremento significativamente mayor que las intervenidas de forma parcial y no hubo nuevos brotes en las cepas control. En el segundo año todos los tratamientos incrementaron su biomasa, aunque sólo fue significativo $(P<0,01)$ en las cepas con cosecha total (figura 6).

El DAP medio de los vástagos remanentes de las cepas con cosecha parcial no presentó diferencias significativas con los vástagos de las cepas control al final del ensayo. Las cepas control mantuvieron un DAP medio de sus vástagos de 2,4 cm durante los dos años de evaluación y los vástagos de las cepas con cosecha parcial presentaron un incremento medio de $0,1 \mathrm{~cm}$ después de los dos años de evaluación, con un DAP medio de 2,9 cm.

El modelo de acumulación de biomasa para los rebrotes presentó un buen ajuste $(P<0,01)$ (figura 5). Durante el primer año, posterior a la cosecha, las cepas con cosecha total y parcial presentaron un incremento de 3,9\% respecto a la biomasa aérea inicial, porcentaje menor al alcanzado por las cepas control que incrementaron su biomasa en 5,3\% (figura 7). Para el segundo año las cepas con cosecha total fueron las que presentaron un mayor incremento anual con un $14 \%$, seguidas por las cepas con cosecha parcial 

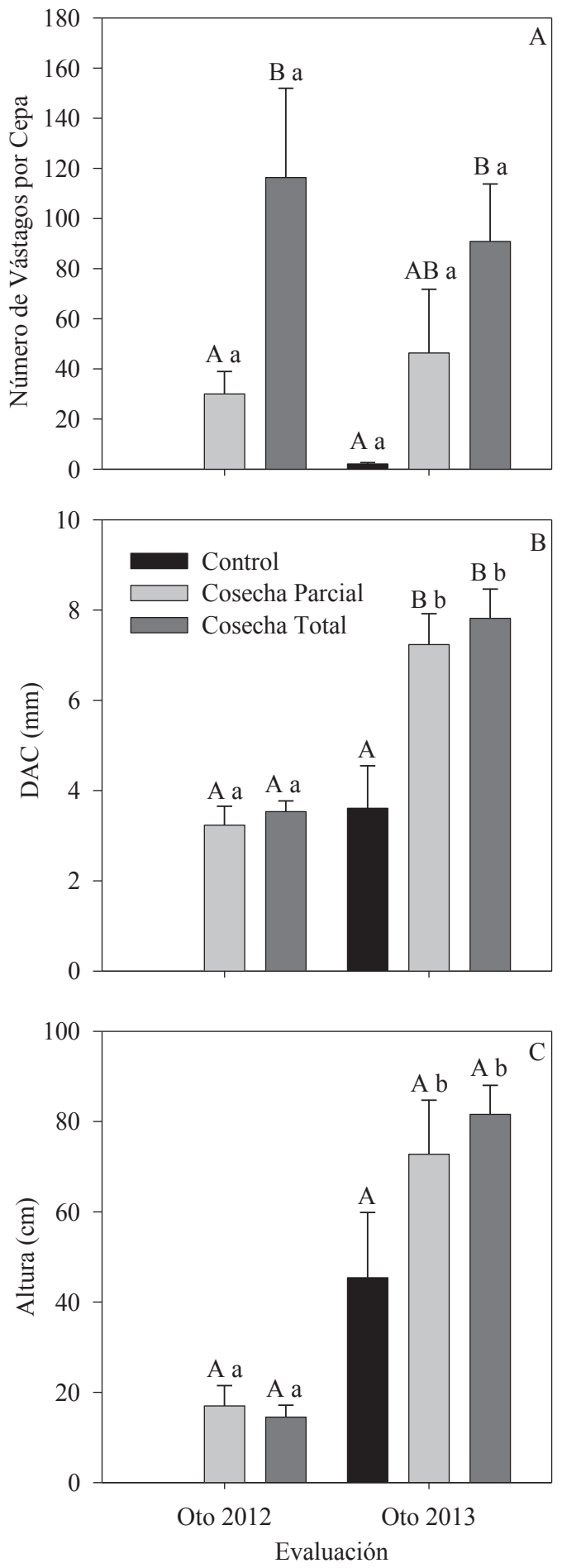

Figura 4. Crecimiento de rebrotes en cepas de Peumus boldus bajo diferentes tratamientos de corta, en el verano de dos años consecutivos. A) Número de rebrotes por cepa, B) diámetro a la altura del cuello (DAC, $\mathrm{cm})$ y C) altura $(\mathrm{cm})$. Las letras mayúsculas indican diferencias significativas entre tratamientos y las minúsculas, entre meses de evaluación (Tukey, $P<0,05$ ).

Sprouts growth of Peumus boldus coppice under different cut treatments, in the summer of two consecutive years. A) Number of shoots per stump, B) root collar diameter (DAC, $\mathrm{cm})$ and C) height (cm). Capital letters indicate significant differences among treatments and lowercase letters indicate differences among months of evaluation (Tukey, $P<0.05$ ).

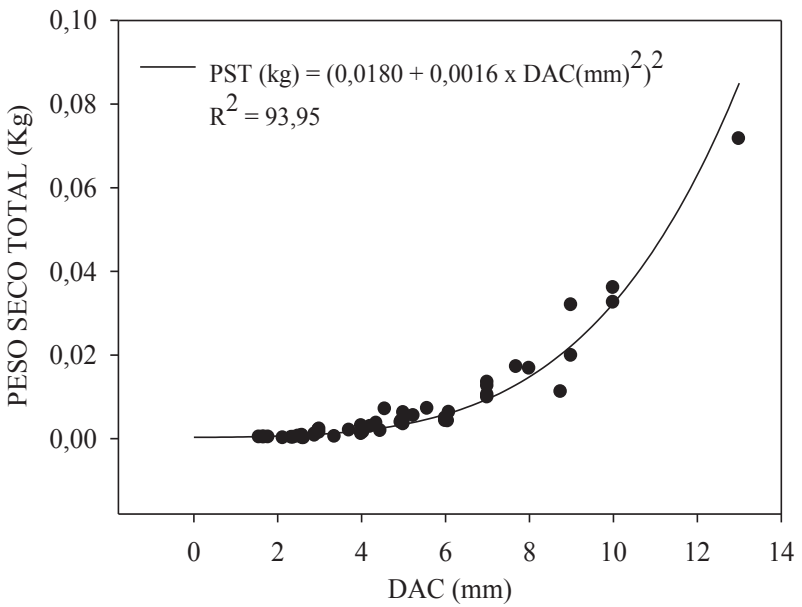

Figura 5. Modelo ajustado de la biomasa de rebrote de Peumus boldus (peso seco total, PST $\mathrm{kg}$ ) en función del diámetro a la altura del cuello (DAC, $\mathrm{mm}$ ).

Adjusted model of Peumus boldus sprouts biomass (total dry weight, PST kg) in function of root collar diameter (RCD, mm).

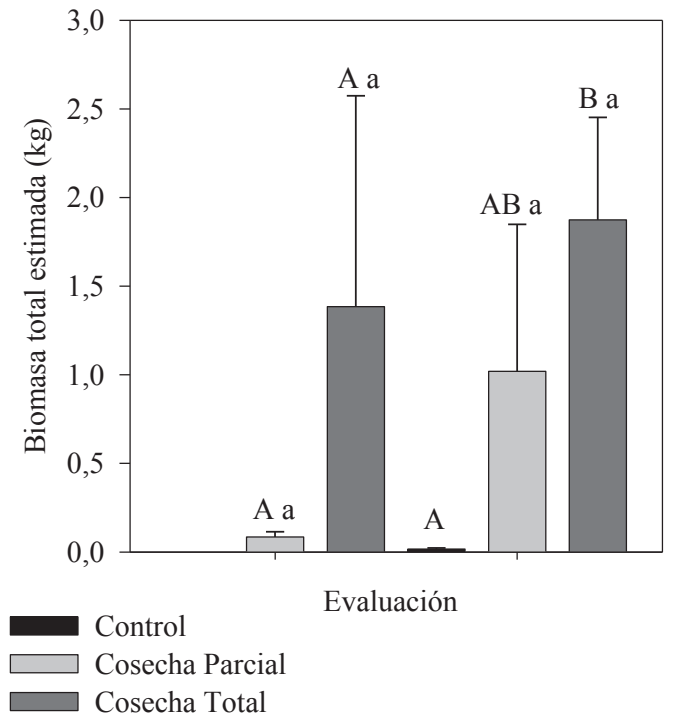

Figura 6. Biomasa de rebrotes de Peumus boldus bajo diferentes tratamientos de corta en el período de evaluación. Las letras mayúsculas indican diferencias significativas entre tratamientos y las minúsculas, entre meses de evaluación (Tukey, $P<0,05$ ).

Peumus boldus sprouts biomass under different treatments of cutting within the evaluation period. Capital letters indicate significant differences among treatments and lowercase letters among months of evaluation (Tukey, $P<0.05$ ).

con $8,3 \%$ y las control con 4,6 \% (figura 7). Al final del periodo de evaluación las cepas con cosecha total habían recuperado un $17,9 \%$ de su biomasa aérea inicial, las con cosecha parcial un 12,2\% y las cepas control tuvieron un incremento acumulado de 9,9\% (figura 7). 


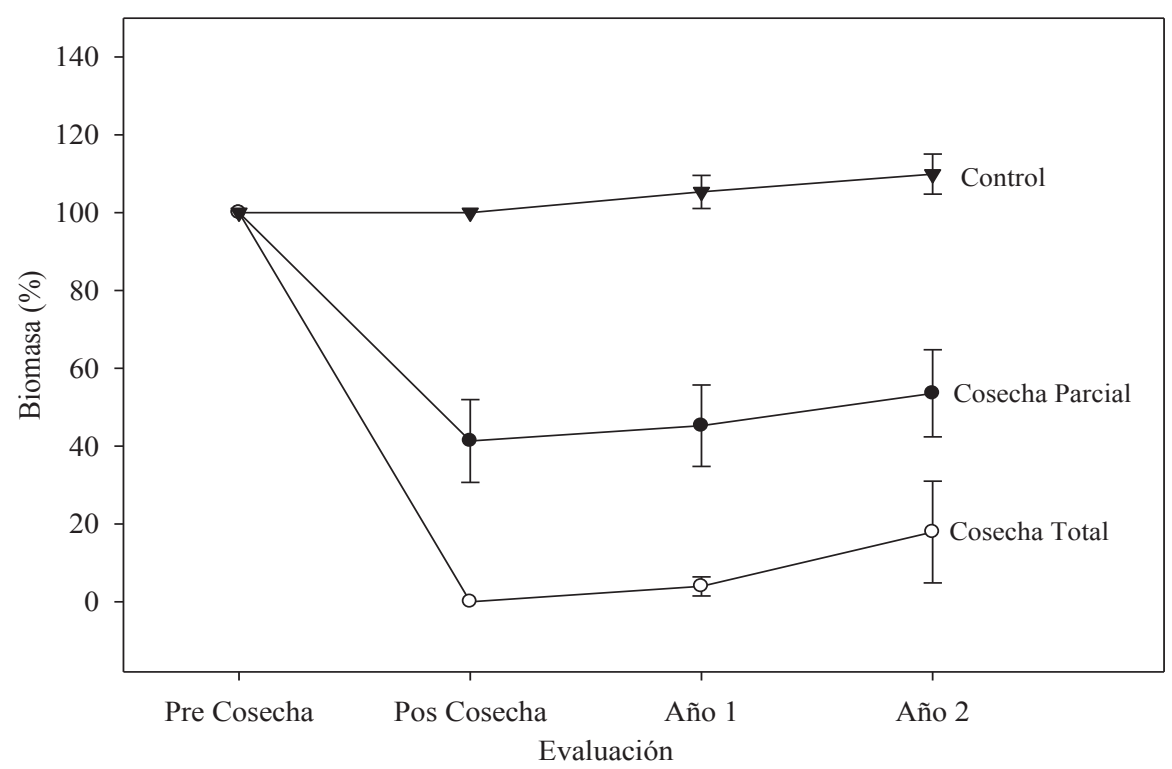

Figura 7. Acumulación de biomasa aérea de Peumus boldus en cepas cosechadas y no cosechadas para un periodo de dos años.

Peumus boldus above-ground biomass accumulation in harvested and unharvested strains within a two-year period.

\section{DISCUSIÓN}

El área de estudio puede considerarse como representativa de estos bosques, ya que los valores de número se cepas de boldo por hectárea se asemejan a los encontrados por Durán (2005), entre 480 y 1.420 cepas ha $^{-1} ; \mathrm{y}$ que el área basal es similar a los valores reportados por Mendoza (2014), entre 9,2 y $12,5 \mathrm{~m}^{2}$ ha $^{-1}$ de área basal, con un rango entre 444 y 627 cepas ha $^{-1}$.

La corta parcial y total de vástagos mejoró la disponibilidad hídrica de los árboles, y a mayor intensidad de la cosecha mejor fue la condición hídrica de la cepa. La menor restricción hídrica de las cepas intervenidas se puede explicar por la disminución de la biomasa aérea que consume agua y la permanencia de gran parte del sistema radical que es capaz de abastecer la demanda hídrica de la biomasa aérea original (Forrester et al. 2012). El mismo efecto se observa al aplicar un raleo en un bosque de Quercus petraea (Matt.) Liebl. donde los árboles raleados presentaron potenciales hídricos más cercanos a cero como consecuencia de la menor tasa relativa de extracción de agua del suelo (Bréda et al. 1995). Además, se suma la capacidad que poseen algunas plantas de desarrollar mecanismos para incrementar la tolerancia a la sequía, que incluyen sistemas radicales bien desarrollados, morfología foliar como hojas esclerófilas o pequeñas (Yin et al. 2005), o la capacidad de realizar ajuste elástico como la que presenta $P$. boldus (Ilabaca 2008). Estas características podrían estar asociadas con los valores de $\Psi$ a (-6,5 MPa) alcanzados por las cepas control.

Los valores de rendimiento cuántico máximo en cepas control e intervenidas estuvieron dentro del rango de 0,00 a
0,85 definido para esta variable (Pérez 2011). El primer año de evaluación estos valores estuvieron bajo el umbral de 0,83 considerado como óptimo, que indicaría que el fotosistema II podría estar sufriendo degradación (Pérez 2011). El segundo año los valores del rendimiento cuántico máximo fueron superiores al estándar óptimo, que indica un buen funcionamiento de los fotosistemas de las hojas evaluadas debido a la mejor condición hídrica con respecto al primer año. Esto se aprecia en las cepas intervenidas donde una mayor disponibilidad de agua permite una mejor respuesta de los procesos fotosintéticos.

Anderson y Andersson (1988) señalan que el rendimiento cuántico máximo está correlacionado con el número de centros funcionales del fotosistema II, por lo que una disminución se considera un indicador de pérdida de función a nivel del aparato fotosintético en respuesta a altas temperaturas y al exceso de radiación, produciendo fotoxidación (Gulías et al. 2002). Aunque también se considera que una disminución de este parámetro se asocia con procesos de disipación de energía por incremento de la disipación térmica o inactivación de centros PSII (Demmig-Adams y Adams 1992). La disminución observada en el rendimiento cuántico máximo durante el primer año de evaluación, podría estar asociada a un daño del aparato fotosintético, pues el rendimiento cuántico no se estaría recuperando durante la noche. Este comportamiento concuerda con lo encontrado por Méthy et al. (1996), quienes señalan que Quercus ilex L. disminuye significativamente el rendimiento cuántico máximo con valores de $\Psi$ a menores a -4,0 MPa. Los valores de $P$. boldus variaron entre $-6,5 \mathrm{MPa}$ en las cepas control y -3,5 MPa en las cepas con cosecha total. Se puede observar una relación directa en- 
tre el $\Psi a$ y rendimiento cuántico máximo, pues la mayor disminución del $\Psi \mathrm{a}$ (-6,5 MPa) en las cepas control genera una mayor disminución en el rendimiento cuántico máximo $(0,7)$, lo que es similar a lo encontrado para $Q$. ilex por Méthy et al (1996), quienes indican que el rendimiento cuántico máximo podría ser cercano a 0,6 cuando el $\Psi$ a se aproxima a -7,0 MPa. Luego, se puede inferir que la intensidad de cosecha tiende a mejorar la respuesta hídrica y fotosintética de P. boldus, pues a mayor intensidad de corta los valores de rendimiento cuántico máximo se encuentran más cercanos al estándar indicado como óptimo y sus valores de $\Psi a$ más cercanos a cero.

En general, valores inferiores a 0,6 de "quenching" fotoquímico de la fluorescencia indican que se está produciendo un proceso de fotoinhibición (Öquist et al. 1992). Este proceso podría estar ocurriendo en las cepas control dado que durante el segundo año mostraron valores medios de 0,36 a diferencia de las cepas intervenidas que estuvieron por sobre dicho umbral ( 0,55 y 0,73 para cosecha total y parcial, respectivamente). Esto evidencia que el raleo parcial de vástagos aumenta la cantidad de energía utilizada en el proceso fotoquímico con la apertura del dosel (Peréz 2011). Por otro lado, la tendencia a la disminución del "quenching" no fotoquímico de la fluorescencia de las cepas con cosecha parcial, evidenciaría una menor disipación térmica, disminuyendo la energía disipada como calor (Gulías et al. 2002, Fleck et al. 2010). Esto concuerda con el aumento de rendimiento cuántico máximo que indica un buen aprovechamiento de la luz y disminuye la necesidad de disipar energía en forma de calor.

Flexas et al. (2014) encontraron que $\Phi$ PSII disminuyó significativamente en $Q$. ilex en verano con respecto al invierno, mientras que el "quenching" no fotoquímico de la fluorescencia presentó valores mayores en invierno. Peumus boldus si bien mostró valores medios de $\Psi$ a inferiores a $-3,5 \mathrm{MPa}$, no mostró diferencias significativas en $\Phi$ PSII. Por otra parte, una disminución en $\Phi$ PSII indica que variables externas generan una caída en la tasa relativa de transporte de electrones (Ogaya y Peñuelas 2003) que también se ve limitado por $\Psi$ a bajos, probablemente debido al cierre de estomas o la inhibición de las reacciones en oscuridad (Méthy et al. 1996). Peña-Rojas et al. (2004) encontraron que una baja en $\Phi$ PSII indica una baja regulación del transporte de electrones, la que fue acompañada por una baja en "quenching" fotoquímico de la fluorescencia, que representa la proporción de centros de reacción del fotosistema II que están abiertos u oxidados. Una situación similar se dio en este estudio con la disminución significativa en "quenching" fotoquímico de la fluorescencia y la tendencia a la baja presentada por $\Phi$ PSII y la tasa relativa de transporte de electrones.

El nulo incremento en DAP de las cepas control y el reducido incremento en los vástagos remanentes de las cepas con cosecha parcial, puede estar asociado a las adversas condiciones que se presentaron los años 2011 y 2012, con un marcado déficit de precipitaciones. Además, Forrester et al. (2012) señalan que la aparición de rebrotes de tocón merma el crecimiento de los vástagos remanentes.

La ausencia de rebrotes en las cepas control puede ser explicada por la alta densidad de cepas y vástagos por hectárea existentes en el bosque, lo que no permitiría la aparición de nuevos brotes por ausencia o falta de luz, ya que la tasa aparición de nuevos brotes se asocia a la intensidad de la intervención (Reyes 2006). Además, el crecimiento y sobrevivencia de los rebrotes está asociado con el tamaño del árbol, el diámetro del tocón, la altura de corte y la relación raíz/rebrote posterior a la corta (Handavu et al. 2011, Singh y Singh 2011). La intensidad de la intervención aplicada produjo una respuesta inmediata en las cepas cosechadas, generando nuevos brotes. En el primer año, el incremento de las cepas con cosecha total fue significativamente superior a las cosechadas de forma parcial. El segundo año se mantuvo la tendencia, pero debido a la alta variabilidad en los datos las diferencias dejaron de ser significativas. La tendencia general es a aumentar, pero es necesario efectuar un monitoreo en un periodo más amplio para evaluar las tasas de acumulación de biomasa.

La intensidad de la intervención está directamente relacionada con el incremento en DAC y altura de los rebrotes. A mayor intensidad de corta, mayor es el incremento de ambas variables. El DAC y la altura de los rebrotes presentaron incrementos significativos entre ambas evaluaciones, que es similar a lo encontrado por Singh y Singh (2011). Además, se ha observado una activa aparición de nuevos brotes posterior a la corta, a partir de lo cual comienza un proceso de competencia y muerte de estos. Del Tredici (2001) señala que después de un daño, el número de brotes que un árbol produce sufre un proceso natural de auto raleo, de tal manera que sólo unos pocos llegarán a convertirse en troncos o vástagos secundarios.

La producción de biomasa de rebrotes está asociada a diferentes variables como: diámetro del tocón, el número y supervivencia de rebrotes, el DAC y el área foliar (Handavu et al. 2011, Singh y Singh 2011). Al final del segundo año la producción de biomasa de $P$. boldus estuvo asociada al incremento en DAC y el número de rebrotes. Sin embargo, también se ha observado en bosque esclerófilo, que la capacidad de rebrote de tocón de Quillaja saponaria Mol. se ha asociado más con la sanidad de la cepa que con al diámetro del tocón (Reyes 2006).

La actual forma de cosecha, que concentra la corta en un subconjunto de cepas, produce una condición hídrica más favorable de estas. Sin embargo, la baja recuperación de la biomasa total previo a la corta, en cepas con cosecha total y parcial al final del segundo año de crecimiento, ponen en duda la capacidad de recuperar la biomasa cosechada en ciclos de corta de 5 años como los actualmente utilizados (Vita 1993). Esto sugiere que la intensidad de cosecha a nivel de cepa debe ser una corta parcial y que esta debe ser inferior a $50 \%$ de área basal. Además, es necesario efectuar estudios que permitan determinar la intensidad y periodicidad de la cosecha. 


\section{AGRADECIMIENTOS}

Este estudio se realizó con el apoyo del Proyecto Fondo de Investigación del Bosque Nativo 052/2010 "Uso sustentable de hojas de boldo y piñones de araucaria". Programa de Bosques Mediterráneos, Facultad de Ciencias Forestales y de la Conservación de la Naturaleza, Universidad de Chile. Se agradece especialmente a los estudiantes que colaboraron en el proceso de toma de muestras y procesamiento.

\section{REFERENCIAS}

Anderson JM, B Andersson. 1988. The dynamic photosynthetic membrane and regulation of solar energy conversion. Trends in Biochemical Sciences 13(9): 351-355.

Bellingham PJ, AD Sparrow. 2000. Resprouting as a life history strategy in woody plant communities. Oikos 82(2): 409-416.

Bréda N, A Granier, G. Aussenac. 1995. Effects of thinning on soil and tree water relations, transpiration and growth in an oak forest (Quercus petraea (Matt.) Liebl.). Tree Physiology 15(5): 295-306.

CIREN (Centro de Información de Recursos Naturales, CL). 1996. Estudio agrológico Región Metropolitana. Descripciones de suelos. Materiales y símbolos. Centro de Información de Recursos Naturales. 464 p. (Publicación No 115).

Del Fierro P. 1998. Experiencia silvicultural del bosque nativo de Chile. Recopilación de antecedentes para 57 especies arbóreas y evaluación de prácticas silviculturales. Santiago, Chile. GTZ-CONAF. 420 p.

Del Tredici P. 2001. Sprouting in temperate trees: a morphological and ecological review. The Botanical Review 67(2): 121-140.

Demmig-Adams B, WW Adams. 1992. Photoprotection and other responses of plants to high light stress. Annual Review of Plant Biology 43(1): 599-626.

DGA (Dirección General de Aguas, CL). 2014. Información Oficial Hidrometeorológica y de Calidad de Aguas en Línea. Consultado 01 jul. 2014. Disponible en http://snia.dga.cl/ BNAConsultas/reportes.

Donoso C. 1989. Colección naturaleza de Chile (Volumen 1): Árboles nativos de Chile. Guía de reconocimiento. Cuarta edición. Valdivia, Chile. Marisa Cuneo Ediciones. 116 p.

Donoso S, L Durán. 2007. Evaluación de la producción y productividad en biomasa aérea de boldo (Peumus boldus Mol.) en un bosque esclerófilo. Ciencia e Investigación Forestal 13(1): 125-136.

Durán L. 2005. Evaluación de la producción y productividad en biomasa aérea de boldo (Peumus boldus Mol.) en un bosque esclerófilo de la comuna de María Pinto, Provincia de Melipilla, Región Metropolitana. Memoria Ingeniería Forestal. Santiago, Chile. Facultad de Ciencias Forestales. Universidad de Chile. 55 p.

Fleck I, K Peña-Rojas, X Aranda. 2010. Mesophyll conductance to $\mathrm{CO}_{2}$ and leaf morphological characteristics under drought stress during Quercus ilex L. resprouting. Annals of Forest Science 67(3): 308.

Flexas J, A Diaz-Espejo, J Gago, A Gallé, J Galmés, J Gulías, H Medrano. 2014. Photosynthetic limitations in Mediterranean plants: a review. Environmental and Experimental
Botany 103: 12-23.

Forrester D, CA Bertram, S Murphy. 2012. Impact of competition from coppicing stumps on the growth of retained trees differs in thinned Eucalyptus globulus and Eucalyptus tricarpa plantations in southeastern Australia. Canadian Journal of Forest Research 42(5): 841-848.

Gulías J, J Flexas, A Abadía, H Medrano. 2002. Photosynthetic responses to water deficit in six Mediterranean sclerophyll species: possible factors explaining the declining distribution of Rhamnus ludovici-salvatoris, an endemic Balearic species. Tree Physiology 22(10): 687-697.

Handavu F, S Syampungani, E Chisanga. 2011. The influence of stump diameter and height on coppicing ability of selected key Miombo woodland tree species of Zambia: A guide for harvesting for charcoal production. Journal of Ecology and the Natural Environment 3(14): 461-468.

Ilabaca D. 2008. Respuestas Ecofisiológicas Desarrolladas por Peumus boldus Mol. Frente a Condiciones de Restricción Hídrica. Memoria Ingeniería Forestal. Santiago, Chile. Facultad de Ciencias Forestales. Universidad de Chile. 32 p.

López BC, CA Gracia, S Sabate, T Keenan. 2009. Assessing the resilience of Mediterranean holm oaks to disturbances using selective thinning. Acta Oecologica 35(6): 849-854.

Mendoza E. 2014. Estimación de la cosecha de hojas de boldo (Peumus boldus mol.) Mediante funciones de biomasa en un predio de la comuna de Las Cabras. Memoria Ingeniería Forestal. Santiago, Chile. Facultad de Ciencias Forestales. Universidad de Chile. 31 p.

Méthy M, C Damesin, S Rambal. 1996. Drought and photosystem II activity in two Mediterranean oaks. Annales des Sciences Forestières 53(2-3): 255-262.

Ogaya R, J Peñuelas. 2003. Comparative seasonal gas exchange and chlorophyll fluorescence of two dominant woody species in a Holm Oak Forest. Flora 198(2): 132-141.

Ogaya R, J Peñuelas, J Martinez-Vilalta, M Mangirón. 2003. Effect of drought on diameter increment of Quercus ilex, Phillyrea latifolia, and Arbutus unedo in a holm oak forest of NE Spain. Forest Ecology and Management 180(1): 175-184.

Öquist G, JM Anderson, S McCaffery, WS Chow. 1992. Mechanistic differences in photoinhibition of sun and shade plants. Planta 188(3): 422-431.

Paula S, F Ojeda. 2006. Resistance of three co-occurring resprouter Erica species to highly frequent disturbance. Plant Ecology 183(2): 329-336.

Peña-Rojas K, X Aranda, I Fleck. 2004. Stomatal limitation to $\mathrm{CO}_{2}$ assimilation and down-regulation of photosynthesis in Quercus ilex resprouts in response to slowly imposed drought. Tree Physiology 24(7): 813-822.

Pérez I. 2011. Respuesta fotosintética y bioquímica del follaje de Nothofagus macrocarpa (A. DC.) FM. Vásquez et RA. Rodr. ante un raleo en el cerro El Roble. Tesis Ingeniería Forestal. Santiago, Chile. Facultad de Ciencias Forestales. Universidad de Chile. 37 p.

Reyes M. 2006. Caracterización del desarrollo de rebrotes de diferentes edades, en un monte bajo clareado de quillay ( $Q u i-$ llaja saponaria Mol.), en el secano interior de la IV región. Memoria Ingeniería Forestal. Santiago, Chile. Facultad de Ciencias Forestales, Universidad de Chile. 55 p.

Rodríguez R, O Mattei, M Quezada. 1983. Flora arbórea de Chile. Concepción, Chile. Editorial de la Universidad de Concepción. 408 p. 
Salk CF, SM McMahon. 2011. Ecological and environmental factors constrain sprouting ability in tropical trees. Oecologia 166(2): 485-492.

Singh TC, EJ Singh. 2011. Sprouting shoot biomass dynamics associated with traditional fire in the oak forest stand of Manipur, North East India. Current Science 100(4): 542-547.

Uribe J, R Cabrera, A de la Fuente, M Paneque. 2012. Atlas Bioclimático de Chile. Santiago de Chile, Universidad de Chile. $224 \mathrm{p}$.

Vesk PA. 2006. Plant size and resprouting ability: trading tolerance and avoidance of damage? Journal of Ecology 94(5):
1027-1034.

Vilà M, J Sardans. 1999. Plant competition in mediterranean-type vegetation. Journal of Vegetation Science 10(2): 281-294.

Vita A. 1993. Ecosistemas de bosques y matorrales mediterráneos y sus tratamientos silviculturales en Chile. Investigación y Desarrollo de Áreas Silvestres en Zonas Áridas y Semiáridas de Chile. Segunda edición. Santiago, Chile. CONAF, PNUD, FAO. 235 p. (Documento de Trabajo No 21).

Yin C, Y Peng, R Zang, Y Zhu, C Li. 2005. Adaptative responses of Populus kangdingensis to drought stress. Physiologia Plantarum 123(4): 445-451.

Recibido: 29.05 .15

Aceptado: 17.08 .15 